\title{
Dynamic Coverage Verification in Mobile Sensor Networks Via Switched Higher Order Laplacians
}

\author{
Abubakr Muhammad and Ali Jadbabaie \\ Department of Electrical and Systems Engineering \\ University of Pennsylvania, Philadelphia, PA 19104, USA \\ \{abubakr, jadbabai\}@seas.upenn.edu
}

\begin{abstract}
In this paper, we study the problem of verifying dynamic coverage in mobile sensor networks using certain switched linear systems. These switched systems describe the flow of discrete differential forms on time-evolving simplicial complexes. The simplicial complexes model the connectivity of agents in the network, and the homology groups of the simplicial complexes provides information about the coverage properties of the network. Our main result states that the asymptotic stability the switched linear system implies that every point of the domain covered by the mobile sensor nodes is visited infinitely often, hence verifying dynamic coverage. The enabling mathematical technique for this result is the theory of higher order Laplacian operators, which is a generalization of the graph Laplacian used in spectral graph theory and continuous-time consensus problems.
\end{abstract}

\section{INTRODUCTION AND MOTIVATION}

Recent years have witnessed a surge of research interest in science and engineering of networked dynamic systems. Due to the advances in computing, communication, sensing and actuation technologies, networks composed of hundreds or even thousands of inexpensive mobile sensing platforms have become closer to reality. The field of sensor networks is undergoing a revolutionary transformation from a subject of academic curiosity to a mature enabling technology in many industrial and engineering solutions. Although, producing low cost and tiny sensor nodes is still an active area of research, the miniaturization and lowering of cost are understood to follow from recent and future progress in the fields of MEMS and NEMS. Thus, the main challenge in this area has now shifted from the manufacturing of cheap hardware and deployment tools to the development of analytical tools for predicting and controlling the complexities arising in such large-scale networks.

An interdisciplinary research area of network science is emerging which combines disciplines such as communications and networking, graph theory, statistical physics, probability and statistics, social sciences, with robotics, optimization, and control [1]. A major theme in the broad research topic of network science is understanding of the emergence of global behavior from local interactions via adaptation and cooperation. Examples of such phenomena span domains from biological sciences to systems and controls, and from statistical

$\dagger$ This work is supported by DARPA DSO \# HR0011-07-1-0002 via the project SToMP: Sensor Topology \& Minimal Planning, and by ARO MURI grant \# W911NF-05-1-0381. physics to computer graphics, where researchers have been trying to develop an understanding of how a group of moving objects (natural or man-made) can collectively reach a global behavior such as flocking, synchronization or consensus with local interaction rules [2].

A useful abstraction for modeling and analysis of such complex systems has been developed using graph theory. Typically interaction among agents is modeled with graphs in which nodes represent agents and edges represent some form of proximity or other binary relationships [3]. The term graph topology is frequently used to denote the interconnection structure among agents. The use of the term topology (which is typically associated with a branch of mathematics involved with studying sets, the nature of space, its fine structures, and its global properties) is by no means an accident. In fact both disciplines of graph theory and topology where born at the same time, by the famous 1736 paper of Leonard Euler on Seven Bridges of Knigsberg [4], even though algebraic or combinatorial topology became a formal discipline as recent as 1930s [5]. In fact, the very central question of inferring global properties from local information (which is the main goal of research in network science and networked dynamic systems) is the realm of algebraic topology which deals with topics like homology, homotopy, and in general topology of discrete and combinatorial sets.

The philosophy underlying this paper is that meeting the challenges of network science requires a minimalist thinking, i.e. the development of abstractions that retain the essential features of the system using minimum information to manage the explosion of redundancies with scale. We believe that many of these challenges are geometrical and a minimalist approach to solving geometrical problems in networks is essentially topological. In this paper, we use this philosophy to obtain low-cost, provably correct algorithms for the verification of distributed coverage problems in mobile sensing networks.

\section{Problem Formulation}

The primary task of a sensor is to detect, discern or locate certain physical changes within its proximity. In many situations, the environment where such physical changes are expected to take place has such large physical dimensions that it is impossible to monitor it with a single sensor. To accomplish the task of monitoring such an environments, a network of sensors can be deployed in which the individual 
sensor nodes can process and communicate individual sensory data collected in their proximities.

Thus, the first requirement for the deployment of a sensor network is to ensure that the sensors reliably cover the environment in such a way that there are no gaps left in the coverage. Coverage problems for sensor networks have been extensively studied in the literature [6]. When feasible, it is preferred to provide a continual coverage to the environment by deploying a network of static sensing nodes. This is known as the blanket coverage problem. In many cases, it is not feasible to deploy a large number of static sensors for continual coverage. If ubiquitous coverage is not necessary, then one solution is to deploy a mobile sensor network made up of robotic sensing platforms. The mobile agents patrol the environment in such a manner that each point of the environment is visited infinitely often, possibly with a certain frequency. As a result, no point remains undetected for 'too long', as depicted in Figure 1. The problem of verifying such coverage is called a dynamic coverage problem. The dynamic coverage problems have been categorized by Gage in his survey [6] as the sweep and barrier coverage problems. In barrier coverage, the objective is to achieve a static arrangement of elements that minimizes the probability of undetected penetration through the barrier.

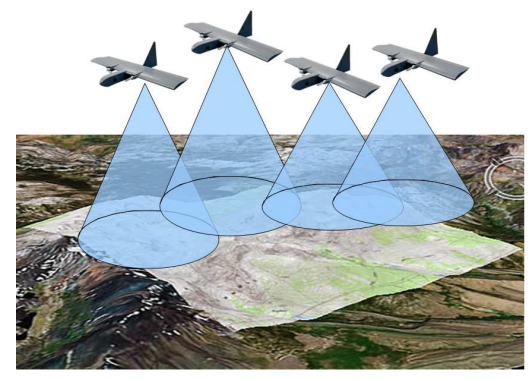

Fig. 1. A dynamic coverage scenario : An area of interest (white) under surveillance by a group of UAV's.

Coverage verification, whether static or dynamic is inherently a geometrical problem and needs some geometrical information about the nodes for computing a solution. Therefore, it is no surprise that many network coverage problems have been studied in the computational geometry literature in various other contexts. For example, the Art Gallery Problem is to determine the number of observers necessary to cover an art gallery (or an area of interest) such that every point in the art gallery is monitored by at least one observer. Inspired by these computational geometry results, many researchers in sensor networks have studied the blanket coverage for static nodes in various settings [7, 8, 9, 10]. In [7], the authors have studied $k$ coverage: whether every point in the service area of the sensor network is covered by at least $k$ number of sensors. In [11, 12], the authors consider network coverage using wireless sensors that conserve power by alternating between active and sleep states with an average sleep period (much) longer than the active period. Other researchers have also examined the area coverage of random sensor networks in bounded domains [13].

The problem of dynamically covering an area of interest has been another subject of interest for various researchers in control theory, networks and robotics. One aspect of bringing mobility into the picture is to design trajectories for network reconfiguration in changing environments or in situations where some areas of the environment are of more interest than others $[14,15,16,17]$. The coverage provided by the network is still blanket, and the mobility of the nodes only cater to a changing surveillance domain. The dynamic coverage problems, as described above, are the sweep and barrier coverage problems. A variant of sweep coverage has been studied under the title of effective coverage for mobile agents in [18]. In [19], sweep coverage schemes have been proposed and studied using a frequency coverage metric that measures the frequency at which every-point in the environment is visited. Similarly, barrier coverage has been studied [20], although for static sensors only, but the results can be extended to studying a dynamic barrier [6].

Such geometrical approaches often suffer from the drawback that they are too expensive to compute in real-time. Moreover, they typically require exact knowledge of the locations of the sensing nodes. Although, this information can be made available in real-time by a localization algorithm or by fitting the sensors with localization sensors (such as GPS), it can only be used most effectively in an off-line pre-deployment analysis for large networks or when there are strong assumptions about the geometrical structure of the network and the environment. Due to the challenges in designing effective distributed algorithms, there may be a massive accumulation of information at a small subset of nodes. Furthermore, most computational geometry algorithms suffer from a lack of robustness due to the required precision in exact geometrical information. In addition, if the network topology changes due to node mobility or node failure, a continuous monitoring of the network coverage becomes prohibitive if the algorithm is too expensive to run or is sensitive to location uncertainty. Finally, localization equipment adds to the cost of the network.

To address these issues, we use a fresh approach for distributed coverage verification using minimal geometry. Our goal here is to show that surprisingly simple, and minimal topological information, such as the ID's of nearest neighbors can be used to this end. The geometrical information that is needed for solving such problems is remarkably minimal: no means of measuring distance, orientation, or location of the nodes in an environment are required. As described below, the basic idea is to infer geometric proximity of other nodes by the mere existence of radio communication (without any aid of specialized localization equipment or refinement in proximity). From this connectivity data alone, coverage for the entire network is verifiable using the tools of algebraic topology.

Our approach is quite interdisciplinary in nature and combines recent advances in multiagent systems and control 
on agreement and consensus problems [2, 21], with recent advances in coverage maintenance in sensor networks using computational algebraic topology methods [22, 23, 24, 25]. to develop distributed algorithms that are computationally inexpensive and robust to failures.

\section{BEyOND GRAPHS: Simplicial Models OF NETWORKS}

\section{A. From graphs to simplicial complexes}

Graphs can be generalized to more expressive combinatorial objects known as a simplicial complexes. For a thorough treatment of simplicial complexes and their topological invariants, see for example [26]. While graphs model binary relations, simplicial complexes can be used to model higher order relationships. Given a set of points $V$, a $k$-simplex is an unordered set $\left\{v_{0}, v_{1}, \cdots, v_{k}\right\} \subset V$ such that $v_{i} \neq v_{j}$ for all $i \neq j$. The faces of the $k$-simplex $\left\{v_{0}, v_{1}, \cdots, v_{k}\right\}$ are defined as all the $(k-1)$-simplices of the form $\left\{v_{0}, \cdots, v_{j-1}, v_{j+1}, \cdots, v_{k}\right\}$ with $0 \leq j \leq k$. A simplicial complex is a collection of simplices which is closed with respect to the inclusion of faces. One can define an orientation for a simplicial complex by defining an ordering on all of its $k$-simplices. We denote the $k$-simplex $\left\{v_{0}, \cdots, v_{k}\right\}$ with an ordering by $\left[v_{0}, \cdots, v_{k}\right]$, where a change in the orientation corresponds to a change in the sign of the coefficient as $\left[v_{0}, \cdots, v_{i}, \cdots, v_{j} \cdots, v_{k}\right]=$ $-\left[v_{0}, \cdots, v_{j}, \cdots, v_{i} \cdots, v_{k}\right]$.

With these definitions, it is clear that a graph is a simplicial complex which its vertices and edges are in fact the 0- and 1 -simplices respectively, and each vertex is a face of all its incident edges. An oriented simplicial complex is merely a generalization of a directed graph ${ }^{1}$

One can also generalize the concepts of adjacency and degree to simplicial complexes. Two $k$-simplices $\sigma_{i}$ and $\sigma_{j}$ are upper adjacent (denoted by $\sigma_{i} \frown \sigma_{j}$ ) if both are faces of a $(k+1)$-simplex in $X$. The two $k$-simplices are said to be lower adjacent (denoted by $\sigma_{i} \smile \sigma_{j}$ ) if both have a common face (see Figure 2 below).

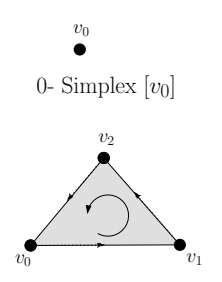

2- Simplex $\left[v_{0}, v_{1}, v_{2}\right]$

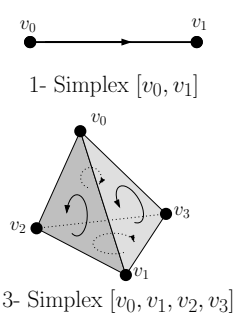

Fig. 2. (Left:) Examples of 1,2, and 3 simplices. (Top Right): Two nodes that are connected by an edge; two edges that have a common face are upper adjacent. (Bottom Right) Two faces that share an edge and two tetrahedra that share a face are lower adjacent.

Having defined the adjacency, one can define the upper and lower adjacency matrices, $A_{u}$ and $A_{l}$ respectively, in a similar

\footnotetext{
${ }^{1}$ Note that this should not be confused with the notion of a hyper graph in which any subset of the power set of vertices could correspond to a hyper edge.
}
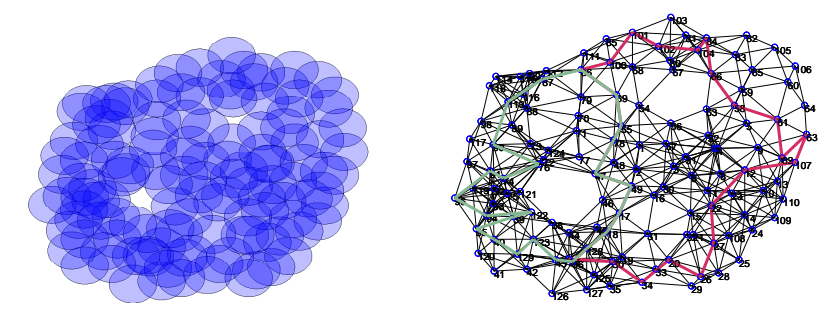

Fig. 3. (Left) Sensor coverage with disk footprints.(Right) The corresponding Čech complex. Coverage holes are represented by holes in the Čech complex.

fashion to a graph's adjacency matrix. The upper and lower degree matrices $D_{u}$ and $D_{l}$ are also defined similarly.

If $X$ is a finite simplicial complex, for each $k \geq 0$, define $C_{k}(X)$ to be the vector space whose basis is the set of oriented $k$-simplices of $X$. We let $C_{k}(X)=0$, if $k$ is larger than the dimension of $X$. Each element of these vector spaces is a linear combination of the basis elements. Over these vector spaces the $k$-th boundary map is defined to be the linear transformation $\partial_{k}: C_{k}(X) \rightarrow C_{k-1}(X)$, which acts on the basis elements of its domain via

$$
\partial_{k}\left[v_{0}, \cdots, v_{k}\right]=\sum_{j=0}^{k}(-1)^{j}\left[v_{0}, \cdots, v_{j-1}, v_{j+1}, \cdots, v_{k}\right] .
$$

Intuitively, the boundary map $\partial_{k}$ operated on a $k$-simplex, returns a linear combination of its $k+1$ faces. In fact the zeroth boundary map $\partial_{1}$ is nothing but the edge-vertex incidence matrix of a graph which maps edges (1 simplices) to nodes (0 simplices), mapping a simplex to its boundary (hence the name boundary map).

Using (1), it is easy to show that the composition $\partial_{k} \circ \partial_{k-1}$ is uniformly zero for all $k$ and as a result, we have $\operatorname{Im} \partial_{k} \subset$ $\operatorname{Ker} \partial_{k-1}$. Then, the $k$-th homology group of $X$ is defined as

$$
H_{k}(X)=\operatorname{Ker} \partial_{k-1} / \operatorname{Im} \partial_{k} .
$$

Since the boundary operators $\partial_{i}$ map a simplex to a linear combination of its faces (i.e. its boundary), the homology groups can be used to distinguish topological spaces from one another. More precisely, the dimension of the $k$-th homology group (known as its Betti number) identifies the number of $k$-dimensional "holes" in the given topological space. For example, the dimension of $H_{0}(X)$ is the number of connected components of the 1-skeleton (or collection of 0 and 1 simplices, i.e., nodes and edges) of $X$. On the other hand, the dimension of $H_{1}(X)$ is the number of its non-contractible ${ }^{2}$ cycles or holes.

Homology groups are used to distinguish topological spaces from one another by identifying the number of 'holes' of various dimension, contained in these spaces. Each nontrivial homology class in a certain dimension helps identify a corresponding hole in that dimension. Crudely speaking, the dimension of $H_{0}(X)$ is the number of connected components (0-dimensional holes) of $X$. the dimension of $H_{1}(X)$ is the

\footnotetext{
${ }^{2} \mathrm{~A}$ space is contractible if it is homotopy equivalent to a single point.
} 
number of non-contractable cycles in $X$. For a surface, it identifies the 'punctures' in that surface. For a graph it is the number of loops or circuits. $H_{2}(X)$ identifies the number of 3 -dimensional voids in a space and so on.

\section{B. Čech and Rips complexes and coverage problems}

Since we aim to apply the topological properties of the simplicial complexes to the coverage problem and therefore dealing with union of disks, it would be useful to define what is known as the Čech or Nerve complex. Given a collection of sets $\mathcal{U}=\left\{\mathcal{U}_{i}\right\}$, the Čech complex of $\mathcal{U}$ denoted by $\mathcal{C}(\mathcal{U})$ is the abstract simplicial complex whose $k$-simplices correspond to non-empty intersections of $k+1$ distinct elements of $\mathcal{U}$. This complex is simply formed by associating convex sets (e.g. disks) to each node, and then representing edges or higher order simplices from the overlap of such sets. For example, given a network of sensors with disk footprints, we can construct the Čech complex(also known as the nerve complex) by book-keeping over lap of sensor footprints. In the complex, nodes or zero simplices represent the sensors. When two footprints overlap, we draw an edge or a 1-simplex between two nodes ( see Figure 3). However, we also keep track of further set overlaps by representing three disk overlaps with faces or filled triangles and 4 set overlaps with tetrahedra and so forth. A closely related complex, known as the RipsVietoris complex (hereafter called the Rips complex), is the natural analog of proximity graphs (also known as connectivity graphs, r-disk graphs or geometric graphs) which have become ubiquitous in networking, control theory and network science research. In such graphs, nodes represent the agents or sensors and edges represent distance proximity. There is an edge between two nodes if they are within a certain distance of each other. A Rips complex is just a generalization of this concept to higher order relations (see Figure 4). If 2 nodes are within the distance of each other, there is an edge between them. When 3 nodes are within a distance of each other then the three nodes will form a face. If four nodes are within a certain distance, they form a tetrahedron. While the Rips complex is the suitable abstraction for modeling nearest neighbor communications, the Čech complex is suitable for coverage problems.
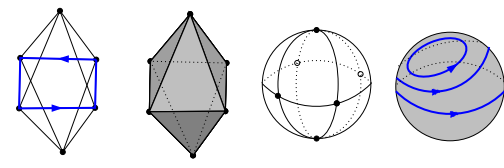

Fig. 4. A connectivity graph [left], its Rips complex [center left] and a depiction of its topology as a sphere [right].

A well-known result in algebraic topology known as the Čech theorem [27] implies that if collection of sets $\mathcal{U}=\left\{\mathcal{U}_{i}\right\}$ and their non-empty finite intersections are contractible (i.e., the sets themselves have no holes and their intersections have no holes) then the Čech complex $\mathcal{C}$ has the same homotopy type as the union of $\mathcal{U}_{i} \mathrm{~s}$. The above result indicates that in order to verify coverage in a given domain by a set of sensors, one only needs to look at the homology groups (which represents holes) of the underlying Čech complex. If the Čech complex has no holes, neither does the sensor cover. However, computation of the Čech complex is not an easy task, as it requires localization of each sensor as well as measurement of distances in order to verify that sensor footprints overlap. Furthermore, the Čech complex is very fragile with respect to uncertainties in distance and location measurements [25]. On the other hand, the Rips complex can be easily formed by merely communication with nearest neighbors, solely based on local connectivity information. However, the Rips complex is unfortunately not rich enough to contain all the topological and geometric information of the Čech complex and in general does not tell us information about coverage holes. Recently, the authors in [22] have shown that in certain cases, the Rips complex does carry the necessary information. Namely, it is shown that any Čech complex $\mathcal{C}_{r_{c}}$ made from sensor footprints of disks of radius $r_{c}$, can be "sandwiched" between two Rips complexes formed from nearest neighbor communication with broadcast disk radius $r_{b 1}=\sqrt{3} r_{c}$ from left and $r_{b 2}=2 r_{c}$ from right. In other words,

$$
\mathcal{R}_{\sqrt{3} r_{c}} \subseteq \mathcal{C}_{r_{c}} \subseteq \mathcal{R}_{2 r_{c}},
$$

where $\mathcal{R}_{\epsilon}$ is a Rips complex formed from broadcast disks of radius $\epsilon$, and $\subseteq$ represents containment of one simplicial complex in another. Therefore, if the Rips complex with broadcast disks of radius $r_{b 1}$ is hole free, then so is the sensor coverage, and if the Rips complex with broadcast radius of $r_{b 2}$ has holes, so does the coverage. In other words, this result gives us a necessary and a a sufficient homological criterion for coverage verification

In principle, one can detect holes in a simplicial complex by looking at the homology groups associated with that complex. However such linear algebra computation are generally centralized [22]. In what follows, we resent our preliminary results on detection of coverage holes in a decentralized fashion. Before doing so, however, we need to introduce the machinery of combinatorial Laplacians for simplicial complexes (which are again generalizations of the same notion for graphs).

\section{Combinatorial Laplacians}

The graph Laplacian [28] has various applications in image segmentation, graph embedding, dimensionality reduction for large data sets, machine learning, and more recently in consensus and agreement problems in distributed control of multi agent systems $[2,21]$. We now define the Laplacian matrix of a graph and its generalizations to simplicial complexes.

If the vertex-by-edge-dimensional matrix $B$ is the incidence matrix of a graph $\mathcal{G}$, then its Laplacian matrix is defined as $L=B B^{T}$. As it is evident from the definition, $L$ is a positive semi-definite matrix. Also it is well-known that the Laplacian matrix can be written in terms of the adjacency and degree matrixes of $\mathcal{G}$ as well:

$$
L=D-A,
$$


which implies that the $i$-th row of the Laplacian matrix only depends on the local interactions between vertex $i$ and its neighbors.

A similar operator can be defined for simplicial complexes using the boundary maps [29]. The operator $\mathcal{L}_{k}: C_{k}(X) \rightarrow$ $C_{k}(X)$ defined as

$$
\mathcal{L}_{k}=\partial_{k+1} \partial_{k+1}^{*}+\partial_{k}^{*} \partial_{k}
$$

is called the $k$-th combinatorial Laplacian of the simplicial complex $X$, where the operators have simple matrix representations and $\partial_{k}^{*}$ is the adjoint operator of $\partial_{k}$. Note that the expression for $\mathcal{L}_{0}$ reduces to the definition of the graph Laplacian matrix. Also similar to the case of graph Laplacian, the combinatorial Laplacians can be represented in terms of the adjacency and degree matrices as follows [30]:

$$
\mathcal{L}_{k}=D_{u}-A_{u}+(k+1) I+A_{l} \quad k>0,
$$

where $I$ is the identity matrix of the proper size, $A_{l}$ is the lower adjacency matrix between $k$-simplices and $A_{u}$ is the corresponding upper adjacency matrix. This equation indicates that $\mathcal{L}_{k}$ is a positive semi-definite matrix, whose kernel represents the cohomology groups ${ }^{3}$ [30]. This is merely extension of the fact that in the case of graphs, the kernel of the Laplacian matrix represents the connected components (0dimensional holes). Moreover, (3) implies that as in the case of the graph Laplacian, the $i$-th row of $\mathcal{L}_{k}$ only depends on the local interactions between the $i$-th $k$-simplex and its upper and lower adjacent simplices. As a result, to verify a hole-free coverage in the plane, one needs to verify that the kernel of the $k$-Laplacian of the Rips complex with radius $r_{b 1}$ above is zero. This property makes the combinatorial Laplacian a suitable tool for distributed coverage verification. In the next section we study dynamic coverage verification based on these and other observations.

\section{Mobile Networks And Dynamic Coverage}

\section{A. Graph Laplacian, Consensus and Homology}

The dimension of the null space of this graph Laplacian is well known to be equal to the the number of connected components of the graph [28]. Therefore, it is no coincidence that $\operatorname{ker} \mathcal{L}_{0} \cong H_{0}(X)$ also counts the number of connected components of $X$. Moreover, when the graph is connected, the vector $\mathbf{1}=\left[\begin{array}{lll}1 & 1 & \ldots 1\end{array}\right]^{T}$ is always an eigenvector of $\mathcal{L}_{0}$ corresponding to the eigenvalue 0 . Again, this can be explained in terms of the dual space to homology, i.e. the cohomology. Note that the zero dimensional cohomology is an equivalence class of functions on the vertices [27]. The vector 1 corresponds to the zero cohomology class of constant functions on the vertices. Any non-zero vector in the span of 1 represents a constant function in this cohomology class. If there are more than one connected components, there is a cohomology class of locally constant functions for each

\footnotetext{
${ }^{3}$ Strictly speaking, cohomology groups are dual spaces of homology groups For the sake of our discussion however, this distinction is immaterial as either one represent $\mathrm{k}$ dimensional holes in the simplicial complex.
}

component [27]. The locally constant functions are precisely the harmonic functions predicted by Hodge theory.

Let us turn our attention to consensus algorithms for multiagent systems that are based on the graph Laplacian. If $x_{i}$ : $v_{i} \rightarrow \mathbb{R}$ is a scalar function on vertex $v_{i}$, then the state vector $\mathbf{x}(t)=\left[x_{1}(t) x_{2}(t) \ldots x_{n}(t)\right]^{T}$ can be used to model the evolution of the system,

$$
\dot{\mathbf{x}}(t)=-\mathcal{L}_{0} \mathbf{x}(t),
$$

in which $\mathcal{L}_{0}$ captures the connectivity between the nodes in the graph. If the underlying graph is connected, then $\lim _{t \rightarrow \infty} \mathbf{x}(t)=c \mathbf{1}$, where $c$ is a constant that depends on $\mathbf{x}(0)$. Thus connectedness is a sufficient condition for consensus.

In [2], the authors have proven consensus under a weaker condition of joint connectedness. A collection of finite graphs is said to be jointly connected if their union is a connected graph. The basic idea is to model the dynamics under changes in the graph topology as a switched linear system by

$$
\dot{\mathbf{x}}(t)=-\mathcal{L}_{0}^{\sigma(t)} \mathbf{x}(t),
$$

where $\sigma:[0, \infty) \rightarrow \mathcal{P}$ is a switching signal that indexes the appropriate graph topology at each switch. The main result in [2] says that consensus is possible even under the (weaker) condition of joint connectedness of the graphs encountered in an infinite sequence of contiguous bounded time-intervals.

Based on these results, one can ask if the consensus dynamics can be generalized to some switched linear systems that models the flow of the $k$-Laplacian under switching topologies. Moreover, if the underlying simplicial complexes are Rips complexes, the topologies change due to the motion of the nodes and one can replace the generalize the concept of joint connectedness to the absence of holes (homology) in certain finite unions of Rips complexes, one can hope to study the sweep coverage problem using the stability properties of that switched linear system. We make these concepts concrete in the following section.

\section{B. k-Laplacian Flows on Simplicial Complexes \\ C. Fixed Network Topology}

The dynamical system (4) runs at the node level in a network. Assume that the nodes are fixed, so that the connectivity graph $G$ of the network is also fixed. Let us now run a dynamical system on the $k$-simplices of a network, as a $k$ Laplacian flow on the corresponding Rips complex $\mathcal{R}$. We described in [30], how to interpret this flow correctly using the language of discrete differential forms. We denote the $k$ forms on a simplicial complex as $C^{k}(\mathcal{R})$, to indicate that they are dual to the chains $C_{k}(\mathcal{R})$. We study the dynamical system

$$
\frac{\partial \omega(t)}{\partial t}=-\mathcal{L}_{k} \omega(t), \quad \omega(0)=\omega_{0} \in C^{k}(\mathcal{R}) .
$$

The equilibrium points of this dynamical system are the set $\operatorname{ker}\left(\mathcal{L}_{k}\right)$. The stability of this dynamical system is described by the following results, proven in [30].

Proposition IV-D: The dynamical system of Equation 5 is semi-stable. 
This implies that the system always converges to to the set $\operatorname{ker}\left(\mathcal{L}_{k}\right)$ in the limit. Note that the condition that $\operatorname{dim} \operatorname{ker}\left(\mathcal{L}_{k}\right)=0$ is equivalent to saying that the $k$-th cohomology group (or the respective $k$-th homology group) is zero. Therefore, we have the following corollary.

Corollary IV-E: [30] The system in Equation 5 is asymptotically stable if and only if $H_{k}(\mathcal{R})=0$.

Thus the asymptotic stability of the system is an indicator of an underlying trivial topology of the complex. These results also prove that for any initial $\omega(0)$, the trajectory $\omega(t), t \geq 0$ always converges to some point in $\operatorname{ker} \mathcal{L}_{k}$. In other words, the dynamical system is a mechanism for producing discrete harmonic $k$-forms on simplicial complexes from any arbitrary $k$-forms [30].

\section{F. Dynamic Network Topology}

Let us now consider the case when the nodes are mobile, giving rise to a switching connectivity graph structure. This also gives rise to a switching structure for the induced Rips complex. Following the presentation in [2], let us write the flow on this changing simplicial complex using a piece-wise constant switching signal $\sigma:[0, \infty) \rightarrow \mathcal{P}$, where $\mathcal{P}$ indexes the set of connectivity graphs on $n$ vertices, emerged by the motion of the nodes. We assume that under reasonable assumptions on the smoothness of the trajectories, there are a finite number of switches in a bounded interval of time. We will further assume that switching periods cannot be smaller than a finite dwell time $\tau_{D}$.

Let the switching times be $t_{1}, t_{2}, \ldots$ If $G_{\sigma\left(t_{0}\right)}, G_{\sigma\left(t_{1}\right)}, \ldots$ are the graphs encountered along a certain evolution, then the corresponding Rips complexes are denoted by $\mathcal{R}\left(G_{\sigma\left(t_{0}\right)}\right), \mathcal{R}\left(G_{\sigma\left(t_{1}\right)}\right), \ldots$, respectively. Let

$$
\mathcal{R}=\bigcup_{i} \mathcal{R}\left(G_{\sigma\left(t_{i}\right)}\right)
$$

Since the Rips complex of a complete graph on $n$ vertices is an $n-1$-simplex, denoted by $\boldsymbol{\Delta}^{n-1}$, it follows that $\mathcal{R} \subseteq$ $\boldsymbol{\Delta}^{n-1}$. We next order the $k$-simplices of $\mathcal{R}$ in the order of their appearance in time and thus produce the spaces $C^{k}(\mathcal{R})$. Thus $C^{k}\left(\mathcal{R}\left(G_{\sigma\left(t_{i}\right)}\right)\right) \subseteq C^{k}(\mathcal{R})$, so the dynamical system

$\frac{\partial \omega(t)}{\partial t}=-\mathcal{L}_{k}^{i} \omega(t), \quad \omega\left(t_{i}\right) \in C^{k}\left(\mathcal{R}\left(G_{\sigma\left(t_{i}\right)}\right)\right), \quad t \in\left[t_{i}, t_{i+1}\right)$,

can also be written as a zero-padded system

$$
\frac{\partial \omega(t)}{\partial t}=-\tilde{\mathcal{L}}_{k}^{i} \omega(t), \quad \omega\left(t_{i}\right) \in C^{k}(\mathcal{R}), \quad t \in\left[t_{i}, t_{i+1}\right) .
$$

Here $\tilde{\mathcal{L}}_{k}^{i}$ is a zero-padded matrix representation of $\mathcal{L}_{k}^{i}$. This lets us write the flow for all $t \geq 0$ as a switched linear system

$$
\frac{\partial \omega(t)}{\partial t}=-\tilde{\mathcal{L}}_{k}^{\sigma} \omega(t), \quad \omega(0) \in C^{k}(\mathcal{R}) .
$$

We want to study the conditions under which this switched linear system is asymptotically stable. From the observation that

$$
\operatorname{ker} \mathcal{L}_{k}^{i} \cong H_{k}\left(\mathcal{R}\left(G_{\sigma\left(t_{i}\right)}\right)\right)
$$

and Corollary IV-E, one can hope that if we are ensured that each simplicial complex encountered during the evolution is hole-free, i.e. $H_{k}\left(\mathcal{R}\left(G_{\sigma\left(t_{i}\right)}\right)\right) \cong 0$, then the switched linear system may show asymptotic stability. We will show below that we can prove the asymptotic stability of the switched system under an even weaker condition, whereby the simplicial complexes encountered in bounded, non-overlapping time intervals are jointly hole-free. The proof of this result closely follows the presentation in [2]. As explained later, this will be a key result in generalizing the previous blanket coverage criteria of $[22,23]$ to a sweeping coverage criterion. Let us give the following definition.

Definition $I V-G$ : Let $\left\{X^{1}, X^{2}, \ldots, X^{m}\right\}$ be a finite collection of simplicial complexes. Then, they are said to be jointly hole-free in dimension $k$ if

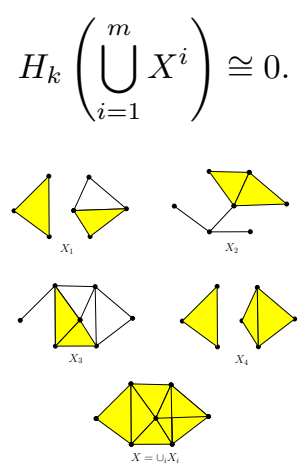

Fig. 5. Jointly hole-free simplicial complexes in dimension 0 and 1. Note that the complexes are not jointly hole-free in dimension 2 due to the hollow tetrahedron in the right part of $X$.

For an example, see Figure 5. We now state the following result:

Proposition $I V-H$ : Let $\mathcal{X}=\left\{X^{1}, X^{2}, \ldots, X^{m}\right\}$ be a finite collection of simplicial complexes, whose union is $X$. Let the $k$-Laplacian operator of $X^{i}$ be given by $\mathcal{L}_{k}^{i}: C^{k}\left(X^{i}\right) \rightarrow$ $C^{k}\left(X^{i}\right)$, which can be zero-padded appropriately to get the operator $\tilde{\mathcal{L}}_{k}^{i}: C^{k}(X) \rightarrow C^{k}(X)$. If $\mathcal{X}$ is jointly hole-free in dimension $k$, then

$$
\bigcap_{i=1}^{m} \operatorname{ker} \tilde{\mathcal{L}}_{k}^{i}=\{0\}
$$

Proof: First note that $\operatorname{ker} \tilde{\mathcal{L}}_{k}^{i} \cong \operatorname{ker} \mathcal{L}_{k}^{i} \oplus F^{i} \cong H_{k}\left(X^{i}\right) \oplus F^{i}$, where $F^{i}$ is the space spanned by the eigenvectors corresponding to the 'fake' zero eigenvalues, due to zero-padding. Therefore, the zero-padding does not present an obstruction in analyzing the intersection of the kernels and is only there for proper comparison. For notational convenience, let us also write $\tilde{\mathcal{L}}_{k}^{i}$ as $\tilde{\mathcal{L}}_{k}\left(X^{i}\right)$. It is easy to see that

$$
\tilde{\mathcal{L}}_{k}\left(X^{p}\right)+\tilde{\mathcal{L}}_{k}\left(X^{q}\right)=\tilde{\mathcal{L}}_{k}\left(X^{p} \cap X^{q}\right)+\tilde{\mathcal{L}}_{k}\left(X^{p} \cup X^{q}\right),
$$

which can be generalized to the relation

$$
\sum_{i=1}^{m} \tilde{\mathcal{L}}_{k}\left(X^{i}\right)=\tilde{\mathcal{L}}_{k}\left(\bigcup_{i=1}^{m} X^{i}\right)+\sum_{i=1}^{m-1} \tilde{\mathcal{L}}_{k}\left(X^{i+1} \cap\left(\bigcup_{j=1}^{i} X^{j}\right)\right) .
$$


Since the zero-padded $k$-Laplacians are all positive semidefinite, a $k$-form $w \in C^{k}(X)$ which is harmonic for each $\tilde{\mathcal{L}}_{k}^{i}$, also satisfies

$$
\tilde{\mathcal{L}}_{k}\left(\bigcup_{i=1}^{m} X^{i}\right) \omega=0
$$

Therefore,

$$
\bigcap_{i=1}^{m} \operatorname{ker} \tilde{\mathcal{L}}_{k}\left(X^{i}\right) \subset \operatorname{ker} \tilde{\mathcal{L}}_{k}\left(\bigcup_{i=1}^{m} X^{i}\right)=H_{k}\left(\bigcup_{i=1}^{m} X^{i}\right) .
$$

Since by the given condition $\mathcal{X}$ is jointly hole-free, it immediately follows that

$$
\bigcap_{i=1}^{m} \operatorname{ker} \tilde{\mathcal{L}}_{k}^{i}=\{0\}
$$

Given the trajectories $\left\{x_{i}(t)\right\}$ of the nodes, the switched linear system (6) for a fixed $k$, captures the evolution of the Rips complexes as well as the $k$-Laplacian flow on them. We can now state the following result, whose proof has been included in the appendix.

Theorem IV-I: The following are equivalent

1) The switched linear system (6) is globally asymptotically stable.

2) There is an infinite sequence of switching times $t_{0}, t_{1}, \ldots$, such that across each interval $\left[t_{j}, t_{j+1}\right)$, the encountered collection of Rips complexes are jointly hole-free in dimension $k$.

\section{Proof:}

Suppose that the switched dynamical system (6) is asymptotically stable. Now, suppose to the contrary that there does not exist a sequence of switching times, such that the complexes encountered during the intervals are jointly hole-free. Then there exists a finite $T$, such that for $t \geq T$ there exists a non-zero $k$-form $\theta$ in the intersection of the kernels of all complexes encountered after $T$. In other words, $\tilde{\mathcal{L}}_{k}^{\sigma} \theta=0$ for $t \geq T$. Without loss of generality pick $T=0$. The evolution of (6) can be written as

$$
\omega(t)=\left(\prod_{i=0}^{\infty} \exp \left(-\tilde{\mathcal{L}}_{k}^{i}\left(t_{i+1}-t_{i}\right)\right)\right) \omega(0) .
$$

Now let $\omega(0)=\theta$, then by virtue of being in the intersection of the kernels, $\omega(t)=\theta$ for all $t \geq 0$. Therefore, we have an initial condition that does not converge to zero. But it is given that the system is asymptotically stable. Therefore, we have a contradiction and the implication is true.

For the converse, Consider the time-interval $\left[t_{j}, t_{j+1}\right)$. By assumption on the switching sequence, there are a finite number of switches in this interval. Let those switching times be given by $t_{j}, t_{j_{1}}, \ldots, t_{j_{N}}$. The Rips complexes encountered during the interval are $\mathcal{R}\left(G_{\sigma\left(t_{j}\right)}\right), \mathcal{R}\left(G_{\sigma\left(t_{j_{1}}\right)}\right), \ldots, \mathcal{R}\left(G_{\sigma\left(t_{j_{N}}\right)}\right)$. By assumption, they are jointly hole-free so that

$$
\operatorname{ker} \tilde{\mathcal{L}}_{k}^{\sigma\left(t_{j}\right)} \cap\left(\bigcap_{i=1}^{N} \operatorname{ker} \tilde{\mathcal{L}}_{k}^{\sigma\left(t_{j_{i}}\right)}\right)=\{0\} .
$$

Now let $\Phi\left(t_{j}, t_{j+1}\right)$ be the product of the individual flows $\exp \left(-\tilde{\mathcal{L}}_{k}^{\sigma\left(t_{j_{m}}\right)}\left(t_{j_{m}+1}-t_{j_{m}}\right)\right)$ between switches, during this interval. By the semi-stability of each individual flow, $\left\|\exp \left(-\tilde{\mathcal{L}}_{k}^{\sigma\left(t_{j_{m}}\right)}\left(t_{j_{m}+1}-t_{j_{m}}\right)\right)\right\| \leq 1$ for each $m$. Therefore, $\left\|\Phi\left(t_{j}, t_{j+1}\right)\right\| \leq 1$.

Let $T$ be an upper bound on the lengths of the intervals $\left[t_{i+1}, t_{i}\right)$, and $M$ be the smallest positive integer such that $M \geq T / \tau_{D}$. Now, by considering all sequences $\mathcal{S}^{M}$ of Rips complexes of length at most $M$ that can occur within an interval of length bounded by $T$, and are jointly-hole free one can define,

$\mu=\max _{\tau_{1} \in\left[\tau_{D}, T\right]} \max _{\tau_{N} \in\left[\tau_{D}, T\right]} \max _{\mathcal{S}^{M}}\left\|\exp \left(-\tilde{\mathcal{L}}_{k}^{s_{1}} \tau_{1}\right) . . \exp \left(-\tilde{\mathcal{L}}_{k}^{s_{N}} \tau_{N}\right)\right\|$.

where $N \leq M$. It can be shown that $\left\|\Phi\left(t_{j}, t_{j+1}\right)\right\| \leq \mu<1$. Furthermore, the repeated application of this inequality across a sequence of intervals yields, $\left\|\Phi\left(t_{p}, t_{p+1}\right) \ldots \Phi\left(t_{1}, t_{2}\right)\right\| \leq$ $\mu^{p}<1$. From these contractions, it is can be inferred that the system is asymptotically stable

\section{J. Dynamic Coverage Verification}

The consequences of this theorem for verifying dynamic coverage are now easy to explain. The property of being jointly hole-free guarantees that in contiguous bounded time-intervals, the union of the Rips complexes has no hole. As mentioned in Section III-B, the topology of the Rips complex can be used to infer blanket coverage (or coverage loss) from network holes. In the same way, the topology of the Rips complexes encountered during the dynamic evolution of a network may be used to infer dynamic coverage of the sensors.

One example is to verify network coverage in which sensors alternate between active and sleep states to conserve power, as depicted in Figure 6. Several constructive schemes have been proposed by various researchers [11, 12, 31, 32, 33, 34]. In order to verify these schemes in real time, we run the dynamical system (6) as a cheap distributed verification method. Since the node are stationary (even though the network topology is changing), the Rips complexes generated during each change in the topology (via wake-ups or sleeping of the nodes) can be compared for the joint hole-free condition. If the Rips complexes are indeed jointly hole-free, then the dynamical system will converge to zero, thus indicating dynamical coverage. Such a scenario has been depicted in Figure 6 .

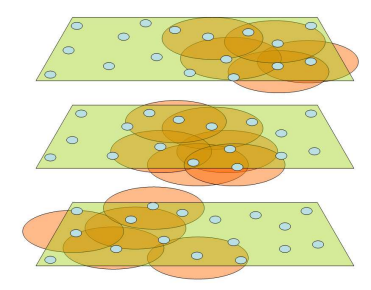

Fig. 6. Sensor wake-up/sleep scheme in a sensor network for providing dynamic coverage of the area under surveillance. 
In the case of dynamic nodes, the above mentioned results are still applicable but the implementation need further work. Due to the changes in node locations, the Rips complexes generated during the evolution cannot be compared directly for a joint hole-free condition. In fact, such a comparison can lead to misleading interpretations of coverage. To fix this, one can use fixed landmarks in the surveillance domain and then compare the complexes in which the landmarks serve as the nodes and the simplices are generated by the visitation of the agents to each landmark. The details of this method will be given in a future work.

Finally, we should mention that our results on dynamic coverage are similar in spirit but quite different in technique to the results on pursuit-evasion problem in [22]. In [22], the authors present a homological criterion for detecting wandering holes in time-varying Rips complexes. The notion of a wandering hole is different from the loss of sweep coverage (one can have sweep coverage with wandering holes). Moreover, our emphasis on decentralized methods results in a technique which is quite different as compared to the method of prism complexes provided in [22].

\section{CONCLUSIONS}

In this paper, we have used the flows of $k$-Laplacian operators on time-varying simplicial complexes for verifying dynamic coverage in mobile sensor networks. The verification has been shown to be equivalent to the asymptotic stability of the switched linear systems that describe those flows. The $k$ Laplacians are a natural generalization of the graph Laplacian. The techniques used for proving consensus under the condition of joint connectedness of switching graphs have been utilized for this coverage problem. This approach gives new insights into the working of consensus algorithms. It also allows us to view algebraic graph theory as a special case of the spectral theory of simplicial complexes. This viewpoint has proven useful in the context of networked sensing and control.

\section{REFERENCES}

[1] M. E. J. Newman, "The structure and function of complex networks," SIAM Review, vol. 45, no. 2, pp. 167-256, 2003.

[2] A. Jadbabaie, J. Lin, and A. S. Morse, "Coordination of groups of mobile autonomous agents using nearest neighbor rules," IEEE Transactions on Automatic Control, vol. 48, no. 6, pp. 988-1001, 2003.

[3] M. Penrose, Random Geometric Graphs. Oxford University Press, 2003.

[4] L. Euler, "Solutio pproblematis ad geometrium situs pertinentis," Commentarii academiae scientiarum petropolitanae, vol. 8, pp. 128-140, 1741.

[5] J. Dieudonné and J. Dieudonné, A History of Algebraic and Differential Topology, 1900-1960. Birkhäuser, 1989.

[6] D. Gage, "Command control for many-robot systems," in Nineteenth Annual AUVS Technical Symposium, pp. 22-24, 1992.

[7] C. F. Huang and Y.-C. Tseng, "The coverage problem in a wireless sensor network," in ACM Intl. Workshop on Wireless Sensor Networks and Applications, 2003.

[8] P. J. W. X. Y. Li and O. Frieder, "Coverage in wireless ad-hoc sensor networks," IEEE Transaction on Computers, vol. 52, no. 6, pp. 753-763, 2003.

[9] B. Liu and D. Towsley, "A study of the coverage of large-scale sensor networks," 2004.

[10] M. P. S. Meguerdichian, F. Koushanfar and M. Srivastava, "Coverage problems in wireless ad-hoc sensor network," in IEEE INFOCOM, pp. 1380-1387, 2001
[11] C. F. Hsin and M. Liu, "Network coverage using low duty-cycled sensors: random and coordinated sleep algorithms," in IPSN, 2004.

[12] F. Ye, G. Zhong, S. Lu, and L. Zhang, "PEAS: a robust energy conserving protocol for long-lived sensor networks," Proceedings of the 10th IEEE International Conference on Network Protocols, pp. 200-201, 2002.

[13] H. Koskinen, "On the coverage of a random sensor network in a bounded domain," in 16th ITC Specialist Seminar, pp. 11-18, 2004.

[14] M. Batalin and G. Sukhatme, "Spreading out: A local approach to multi-robot coverage," Distributed Autonomous Robotic Systems, vol. 5, pp. 373-382, 2002.

[15] M. Batalin and G. Sukhatme, "Sensor coverage using mobile robots and stationary nodes," Procedings of the SPIE, vol. 4868, pp. 269-276.

[16] J. Cortes, S. Martinez, T. Karatas, and F. Bullo, "Coverage control for mobile sensing networks," Robotics and Automation, 2002. Proceedings. ICRA'02. IEEE International Conference on, vol. 2, 2002.

[17] S. Poduri and G. Sukhatme, "Constrained coverage for mobile sensor networks," Robotics and Automation, 2004. Proceedings. ICRA'04. 2004 IEEE International Conference on, vol. 1.

[18] I. Hussein and A. Bloch, "Dynamic coverage optimal control of interferometric imaging spacecraft formations," Decision and Control, 2004. CDC. 43rd IEEE Conference on, vol. 2, 2004.

[19] M. Batalin and G. Sukhatme, "Multi-robot dynamic coverage of a planar bounded environment," IEEE/RSJ International Conference on Intelligent Robots and Systems (Submitted), 2002.

[20] S. Kumar, T. Lai, and A. Arora, "Barrier coverage with wireless sensors," Proceedings of the 11th annual international conference on Mobile computing and networking, pp. 284-298, 2005.

[21] R. Olfati-Saber, J. Fax, and R. Murray, "Consensus and cooperation in networked multi-agent systems," Proceedings of the of IEEE, 2006.

[22] V. de Silva and R. Ghrist, "Coordinate-free coverage in sensor networks with controlled boundaries via homology." To appear in Intl. J. Robotics Research.

[23] V. de Silva and R. Ghrist, "Coverage in sensor networks via persistent homology." To appear in Algebraic and Geometric Topology.

[24] R. G. V. de Silva and A. Muhammad, "Blind swarms for coverage in 2-d," in Robotics: Science and Systems, June 2005.

[25] R. Ghrist and A. Muhammad, "Coverage and hole-detection in sensor networks via homology," in The Fourth International Conference on Information Processing in Sensor Networks, April 2005.

[26] A. Hatcher, Algebraic Topology. Cambridge University Press, 2002.

[27] R. Bott and L. Tu, Differential Forms in Algebraic Topology. SpringerVerlag, Berlin, 1982

[28] R. Merris, "Laplacian matrices of graphs: A survey," Linear Algebra and its Applications, vol. 197, pp. 143-176, 1994.

[29] B. Eckmann, "Harmonische funktionen und randwertaufgaben einem komplex," Commentarii Math. Helvetici, vol. 17, pp. 240-245, 1945.

[30] A. Muhammad and M. Egerstedt, "Control using higher order laplacians in network topologies," in 17th International Symposium on Mathematical Theory of Networks and Systems, 2006.

[31] T. H. L. S. Kumar and J. Balogh, "On k-coverage in a mostly sleeping sensor network," in 10th Intl. Conf. on Mobile Computing and Networking, 2004.

[32] T. He, S. Krishnamurthy, J. Stankovic, T. Abdelzaher, L. Luo, R. Stoleru, T. Yan, L. Gu, J. Hui, and B. Krogh, "Energy-efficient surveillance system using wireless sensor networks," 2004.

[33] M. Nosovic and T. Todd, "Low power rendezvous and RFID wakeup for embedded wireless networks," Annual IEEE Computer Communications Workshop, pp. 3325-3329, 2000.

[34] M. Cardei and J. Wu, "Energy-efficient coverage problems in wireless ad-hoc sensor networks," Computer Communications, vol. 29, no. 4, pp. 413-420, 2006. 